\title{
Statistical Approach To Energy Efficiency Issue In Industrial Pneumatic Systems
}

\author{
Jyrki Parkkinen, Kai Zenger and Joonas Ollila \\ Aalto University, School of Electrical Engineering, \\ Department of Automation and Systems Technology, Helsinki,Finland \\ E-mail: , jyrki.parkkinen@aalto.fi , kai.zenger@aalto.fi, joonas.ollila@aalto.fi ,
}

\begin{abstract}
In this paper, some statistical methods to the energy efficiency issue in industrial pneumatic systems are presented. Since all compressed air (CA) systems are different, in industrial applications it may be difficult to analyse energy efficiency of a single CA system from a theoretical model-based viewpoint. Instead of modeling the industrial pneumatic system from first principles the energy efficiency of the system is analyzed based on time series measurements. The dependency between energy consumption, potential variable pressure and flow variable mass flow makes possible to draw conclusions of the energy efficiency based on the measurements of pressure and power/energy.
\end{abstract}

In woodworking industry, e.g. sawmills, the functioning of the assembly line is relatively monotonic from day to day. Typically, CA is almost entirely used for linear motions. A double acting cylinder is the most common actuator in these applications. The length of the impact, the radius of the piston and radius of the cylinder are the essential key figures of this cylinder type. From these figures and basic dynamics of the cylinder, the air volume per impact can be obtained. When the average values and standard deviation of the air flow in each consumption point (cylinder) is known, statistical methods can be implemented for the total consumption of CA. According to the Central Limit Theorem, the total air consumption, i.e. the sum of consumptions in different consumption points approaches a normal distribution. Since the consumed air flow is proportional to the compressor's energy consumption, the trend of energy can also be approximated as a sum of regression curve and normally distributed deviation component. By this result, a confidence interval for energy consumption can be determined. The result is also ready to be used in energy saving calculations in the future.

Also, the adjustable pressure-difference in a two-point controlled compressor has an essential effect on the overall energy efficiency of the system. When this pressure difference is large, the system is not optimal from the viewpoint of energy efficiency. Typically the time series measurements of pressure are somewhat noisy, but some kind of conclusions of stationary behavior can be done. After possible pre-filtering process of the data, it becomes evident that the measurement data signal is typically a periodic shape with strict minimum and maximum values. The consumption of CA is typically dependent on pressure i.e. higher pressure increases consumption making the shape of pressure curve exponential. A stationary time series described by its mean, variance and autocorrelation function makes possible to identify the essential features of the dynamics of the CA system and the type of consumption.

With the appropriate statistical methods, the results of short-time measurements can be applied for predicting the energy consumption trend in a long run. The combination of first principle modeling with measurements opens new methods in energy efficiency techniques especially when designing new pneumatic systems or the improving of existing designs.

Keywords: Pneumatics in woodworking industry, time series analysis, estimate, confidence interval, autocorrelation 


\section{Introduction}

The subject of the energy efficiency of pneumatic systems has taken a growing interest during the last ten years. Actually, CA systems are major consumers of electric energy and there is plenty of room for improvement. Many comprehensive investigations by research institutes including Fraunhofer[1], University of Cape Town [2] have been made. These investigations list several practical methods to improve energy efficiency in compressed air (CA) systems.

In a comprehensive review on the energy efficiency on pneumatic production [3] several viewpoints on the issue have been taken into consideration. The issue can be approached both energy efficiency use in the generation and treatment of CA and its consumption by end-users (actuators). The analysis of energy efficiency can be based on exergy, i.e. the useful energy, which can be converted to mechanical work. Alternatively, a life cycle costing analysis from product users perspective can be made [3]. Optimization can be made in different levels including pneumatic device and circuit level, condition monitoring and system level.

Also, many theoretical models have been under development lately. In an investigation [4], simulations to form predictive models in industrial applications have been presented. In the model, all types of cylinders, cylinderbased actuators, grippers and blowers are taken into the model. The dynamic modeling approach enables a greater understanding of CA consumption by production machines and lines.

Recently, many simulation models including a Matlab/Simulink model of pneumatic transmission line based on a detailed mathematical descriptions of pneumatic drive components have been developed. In addition to previous models, essential phenomena in CA piping networks like delay and dead time are taken into consideration [5],[6].

For large systems like an industrial pneumatic line, it may also be useful to model the CA system with subsystems. For example, a pneumatic system can be divided into production and consumption subsystems. From the practical viewpoint, since all CA systems are different, in industrial applications it may be difficult to analyze energy efficiency of a single CA system from a theoretical model-based viewpoint since processes involved with the consumption of CA have often stochastic nature.

In this paper, instead of focusing on the models of pneumatic systems, the used method is black-box based. Using measurement data and statistical methods it is relatively simple to form a suggestive general view of the consumption of CA system. The essential parameters affecting the consumption of CA and energy efficiency are the pressure difference of an adjustable two-point compressor.

In his licentiate thesis and paper [7], [8] the author (J.P) has pointed out, that in many industrial pneumatic systems, especially when a two-point compressor is used, the energy efficiency of a CA system can be analyzed based on pressure values. In a simplified form, the duty cycle of a compressor consists of two parts, the compressor is either switched on, when it produces compressed air with full power or it is totally switched off. On the other hand, in some cases, the compressor is still idle running when not producing CA. Typically, in industrial woodworking applications, the idle running power is $20-30 \%$ of the maximum power of the system.

From the viewpoint of energy consumption, the question is the profile of a duty cycle. The pressure difference and the minimum and maximum values of compressor inlet pressure determine the shape and length of the pressure curve. Roughly, the bigger the proportion of the rising time of the total cycle time, the more energy consuming the system.

In practice, an industrial pneumatic system is typically large. The length of a pneumatic line is often hundreds of meters and it has dozens of consumption points. Usually, these consumption points are actuators. The main actuator type in woodworking industry is a double acting cylinder. The dynamics of the air consumption in a single consumption point may be modeled starting from first principles when dimensions of the cylinder are known.

The structure of the paper is as follows. In Ch. 2 the essential factors and features of a typical industrial CA system are classified by their nature. The starting point is the presumption, that the consumption of CA is typically pressure-dependent. In Ch. 3 suitable statistical approach methods for the results are considered. These methods include Central Limit Theorem with an assumption that the sum of regular consumptions approaches a normal distribution. Also, tools for the time series analysis of the pressure measurements are presented. In Ch.4 a case study of a typical woodworking plant in Finland is presented in detail with suggestion for predicting the energy consumption. Ch.5 presents the variables to be measured (pressure, energy) and technical details of the measurement process. Also an interpretation of the measurement results is presented. Final conclusions are presented in Ch.6. 


\section{The factors affecting on energy efficiency of CA system of an assembly line}

In two-point controlled compressor systems, the consumption can be classified in two main types: the pressure-dependent and independent consumption [7] (Fig. $1)$.

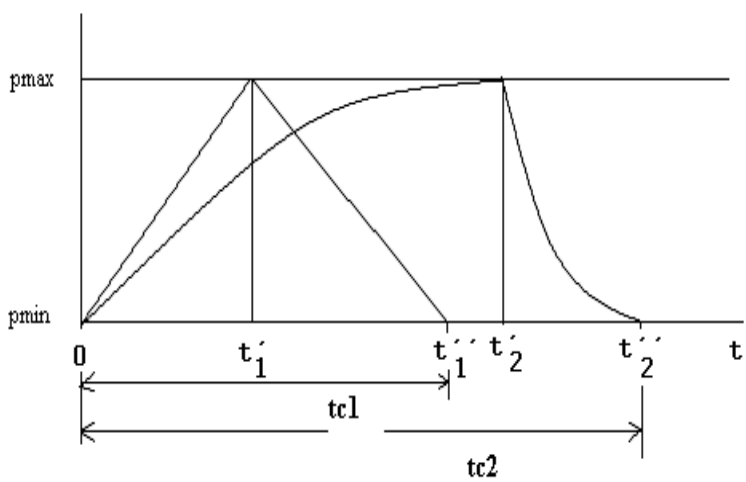

Figure 1: Typical pressure curves in industrial applications in two consumption-types: the pressure-dependent (cycle time $=$ tc2) and pressure-independent (cycle time = tc1) consumptions

The function of the CA network is periodic in both cases. The compressor either works with full when turned on or is switched totally off (2-point control) the volume flow being constant when turned on and 0 when turned off. However, in some applications the compressor is also idle running when not producing CA.

In the case of pressure-independent consumption, the pressure grows linearly when the compressor is switched on and also drops linearly when the compressor is switched off. In that case, it is also possible to determine the consumption of the electrical energy easily.

In practice, the pressure-dependent consumption is much more common than the pressure-independent. In that case, the consumption of compressed air increases as the pressure grows. The pressure profile is an periodical exponential curve between minimum and maximum values. The length of the rise time depends on the adjustable pressure difference.

While basic consumption types in Fig. 1 are ideal consumption models, one can also identify from Fig 2. (measurement of an actual compressor) that the typical consumption is pressure-dependent. Actually, the measurement data of Fig. 2 is smoother than most pressure measurement data series (see Fig.4) and pre-filtering is needed required.

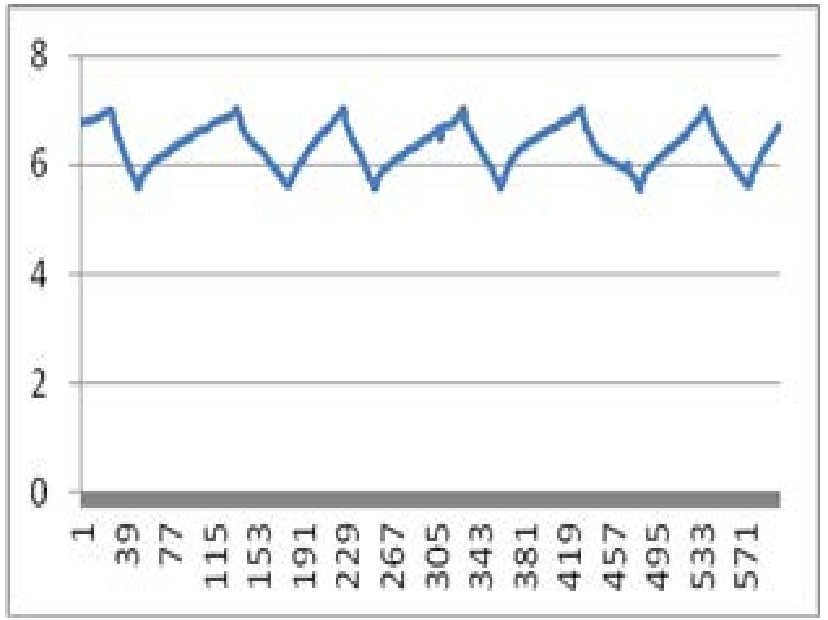

Figure 2: An example of a pressure (bars) time series (duty cycle) of an actual two-point controlled compressor

CA consumption in industry is characteristically less regular. The after treatment and leaks cause pressure losses between production and consumption points.

In the energy efficiency analysis of a manufacturing/assembly line system, it is reasonable to classify parameters by their nature. The parameters can be identified of constant (nominal scale), adjustable and statistical nature. The parameters affecting on the consumption of the CA in a sawmill system in production and consumption by nature are dealt in detail in Chapter 4 and listed in the tables 1 and 2.

The factors that have an effect on the consumption of the CA in a system, can be classified on the factors in production and consumption.

\subsection{Production of CA}

From a purely statistical viewpoint, the units of production systems are of nominal scale and they cannot be changed. Such factors are for example the type of compressor, number and type of filters and type of after treatment system. Usually, the pressure loss caused by a filter type are well known. In a typical CA system, the total pressure losses in the after treatment system are [7]

$\begin{array}{ll}\text { - } & \text { Drier 0.1-0.5 bar } \\ \text { - } & \text { Prefilter } 0.35 \text { bar } \\ \text { - } & \text { Dine filter 0.35 bar } \\ \text { - } & \text { After cooler and piping } 0.2 \text { bar }\end{array}$

On the other hand, many parameters, especially the pressure difference of the compressor can be adjusted to satisfy the consumption of CA optimally. Typically the pressure difference is about 0.3-1 bar. The control of a compressor can also be changed by user (two-point/inverted control) 
and may have a major effect on the consumption of compressed air.

\subsection{Consumption of CA}

In many industrial applications, the consumption points of CA have many similarities. Industrial systems are typically transport systems of bulk material. For example in sawmills, the assembly line starts from the debarking unit and continues via one or more sawing phases. Typically most of these phases have cylinder-based actuators and the operation of the line is quite similar from day to day.

The three main types of cylinders are single-acting, doubleacting and rotating cylinder. In a typical assembly line, the double-acting cylinder is the dominating actuator. The consumption of CA in a single consumption point can be determined from the mechanics and dimensions of the cylinder.

\section{Statistical methods to be applied}

\subsection{Central Limit Theorem}

In probability theory, the Central Limit Theorem (CLT) states that, given certain conditions, the mean of a sufficiently large number of independent random variables, each with a well-defined mean and well-defined variance, approaches a normal distribution. According to the Central Limit Theorem, independent, identically distributed random variables having mean $\mu$ and nonzero variance $\sigma^{2}$, for the sample variance $s_{n}$

$$
\lim _{n \rightarrow \infty} P\left(\frac{s_{n}-n \mu}{\sigma \sqrt{n}} \leq x\right)=\Phi(x)
$$

in which $\Phi(\mathrm{x})$ is the probability that a standard normal random variable is less than $\mathrm{x}$.

\subsubsection{Key figures of a double acting cylinder}

In a typical industrial system, the actuator is a double acting cylinder. The piston motion cycle consist of two motions , the piston first moves to the +-direction consuming air according to the equation

$$
\pi R^{2} s(1+\Pi)
$$

in which

length of the impact $=\mathrm{s}$,

radius of the piston $=\mathrm{r}$,

Radius of the cylinder $=\mathrm{R}$.

For the relative pressure notion $\mathrm{p} / \mathrm{p}_{0}$ notation $\Pi$ is used

and returns to the - -direction when the air consumption is

$\pi\left(R^{2}-r^{2}\right) s(1+\Pi)$

making the total air consumption as a sum of (2) and (3)

$$
\pi s(1+\Pi)\left(2 R^{2}-r^{2}\right)
$$

The consumption rate per time unit can be further obtained from (4) by observing the actuator. If the number of cylinder movements per minute is $\mathrm{N}$, the air volume consumed by the cylinder per time unit is

$$
\mathrm{q}=\mathrm{N} \pi \mathrm{s}(1+\Pi)\left(2 R^{2}-r^{2}\right) / t
$$

For example if $\mathrm{t}=60 \mathrm{~min}, \mathrm{~N}=100, \mathrm{~s}=50 \mathrm{~cm}, \mathrm{R}=10 \mathrm{~cm}, \mathrm{r}$ $=2 \mathrm{~cm}, \mathrm{p}=9 \mathrm{bar}$, makes the total air consumption of 0.5 normal cubic meters per minute.

The consumptions of other cylinder-like actuators can be determined in a similar fashion when the dimensions are known.

\subsubsection{CLT applied to the industrial pneumatic system}

From the basic structure and key figures of the double acting cylinder, it can be easily observed that the air consumed in a impact of the cylinder can be characterized by a welldefined mean and variance. In the common form of the theorem, the random variables are independent and identically distributed (i.i.d), but the theorem can also be used when they are not.

For example, in industrial applications, the variables related to the cylinders may vary according to the size of cylinder. Usually the length of the impact is full, but in some cases may also be incomplete (See also Ch. 5). 
The air consumption of cylinders is predictable, near constant in most consumption points. This makes the variance in most consumption points small resulting that the variance of the sum of consumptions is also small.

If $\mathrm{M}$ is the number of consumption points with an actuator (cylinder), the total consumed volume flow $\mathrm{Q}$ is

$$
Q=\sum_{1=1}^{M} q_{i}
$$

in which air consumption of cylinder $i=q_{i}$

Since one cylinder consumes the same amount of CA per impact, the consumption of CA per time unit can be obtained by observing how many impacts per time unit the cylinder moves.

The behavior of the assembly line is quite stationary from hour to hour. Since energy consumption is strongly, almost linearly dependent on the volume flow, it is therefore reasonable to assume that the trend of the cumulative energy consumption is linear.

Also, it is possible to include stochastic consumptions (blowing purposes) to the model. However, usually the role of these consumptions is minor and they have no significant effect on the overall consumption trend.

\subsection{Time series analysis of pressure measurements}

As can be seen from Fig. 4, the actual pressure curve is far from ideal models in Fig. 1-2. From Fig.4 only the essential figures (pressure difference, $\min / \max$ pressures) can be easily identified.

It is seen that the curve includes noise, which makes the determination of the duty cycle much more elaborate than in the cases shown in Fig. 2. However, since off-line analysis is possible, a practical method for the estimation of the (changing) period and (changing) duty cycle can be described as follows. The minimum and maximum points in the time series sequence are determined by considering continuously the sign between two successive data points. The change negative to positive means minimum and the change positive to negative means maximum. The period is the time between two minima, and the duty cycle is the time between minimum and maximum divided by the period.

To prevent clear mistakes caused by the noisy signal it is possible to introduce security checks by using the standard deviation for example to give the minimum limits to minimum and maximum.

\section{Case study}

On the following, a typical example of a industrial pneumatic system is presented. The system is a woodworking plant (sawmill) in northern Finland. The products of the sawmill include building materials, dimensionally accurate boards and planks and lining material for various purposes. The material of the products is pine.

CA of the sawmill line is produced by three compressors. The compressors are oil-injected two-point controlled screw compressors. The compressors produce about 10, 4 and 3 normal cubic meters of CA in a minute respectively. Usually, inlet power of compressor is $6-7 \mathrm{~kW}$ per normal cubic meter making the powers of compressors about 70, 30 and $20 \mathrm{~kW}$. The energy needed to produce the necessary CA usually depends on the outlet pressure and adjustable pressure-difference of the compressors.

The total number of cylinder-based actuators in the system is about 300 , while the number of working stages is about 100. Almost all of the cylinders are double-acting. Usually almost all these actuators are on use at the same time, the total CA consumption on the assembly line can be obtained by summing these consumptions. Usually, the cylinders are supplied from the same manufacturer to make the maintenance simpler.

Beside actuators, a small portion of CA is used for blowing and other random purposes. It is noteworthy, that in the system there are tanks with a magnitude of $1 \mathrm{~m}^{3}$ for CA soon after compressors. The tanks act like an integrator, which filters the peaks in the consumption. Random consumptions are thus not noticeable.

Table 1: Compressor parameters affecting on the consumption of CA (production)

\begin{tabular}{|l|l|l|l|}
\hline & $\begin{array}{l}\text { Constant/ } \\
\text { Class- } \\
\text { scaled }\end{array}$ & Adjustable & Statistical \\
\hline Idle running power & $\mathrm{x}$ & $\mathrm{x}$ & \\
\hline $\begin{array}{l}\text { Type of the } \\
\text { compressor }\end{array}$ & $\mathrm{x}$ & & \\
\hline
\end{tabular}




\begin{tabular}{|l|l|l|l|}
\hline $\begin{array}{l}\text { Filters of after } \\
\text { treatment system }\end{array}$ & $\mathrm{X}$ & $\mathrm{X}$ \\
\hline $\begin{array}{l}\text { Control/regulation } \\
\text { method of the } \\
\text { compressor }\end{array}$ & $\mathrm{X}$ & $\mathrm{x}$ & \\
\hline $\begin{array}{l}\text { Pressure difference } \\
\text { of the compressor }\end{array}$ & & $\mathrm{x}$ & \\
\hline
\end{tabular}

Typical adjustable parameters of the system (Table 2) are the velocity of the assembly line and the feeding rate of the system. Actually, the feeding rate is usually constant.

The first phases of the assembly line, mainly barking unit are more of stochastic nature than other parts of the line. The typical statistical factor is the size of a trunk. Key figures are the length and the diameter of a trunk. Profile of a typical stem is presented in figure 3 . The length and diameter of a trunk may vary considerably depending on the cargo of wood. Total CA consumption in barking unit can be estimated when information of wood and from figure 3 are available.

Also it is possible that on the same sawmill, the species of tree varies from day to day. Different species of trees have different properties [11]. However, on the case study plant, only pine was used.

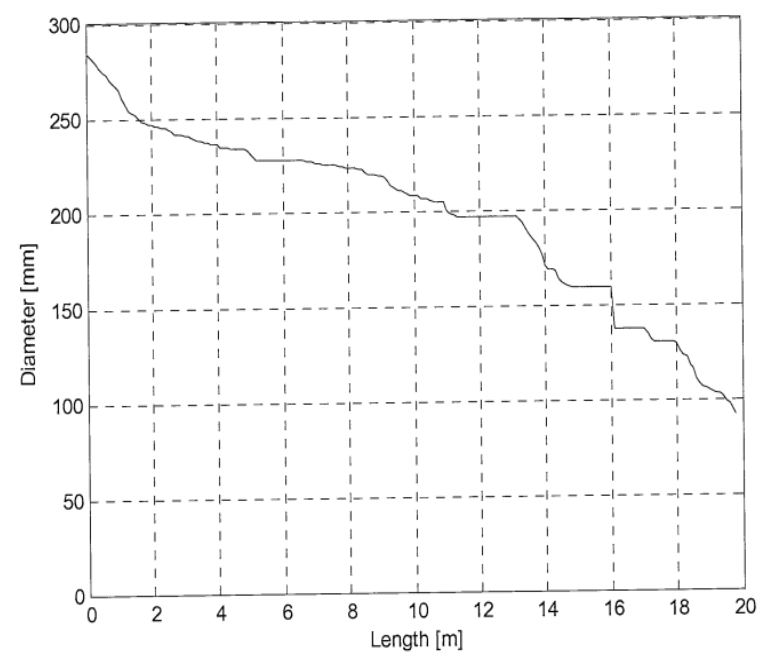

Figure 3: Typical diameter profile of a trunk [11]

Usually, a large trunk effects on the length of the impact of the cylinder, which is in turn proportional to the air consumption. After the debarking unit, the trees typically go through several sawing phases. In these phases the cylinders typically work predictably with full impact.
In most phases, the type of actuator is a double acting cylinder. Also, in a couple of working phases, when movement of the load is done to the upward direction, a single acting cylinder is used.

Table 2: System parameters affecting on the consumption of $C A$ in a woodworking plant (consumption)

\begin{tabular}{|l|l|l|l|}
\hline & $\begin{array}{l}\text { Constant/Class- } \\
\text { scaled }\end{array}$ & Adjustable & Statistical \\
\hline $\begin{array}{l}\text { Species of a } \\
\text { tree }\end{array}$ & $\mathrm{x}$ & $\mathrm{x}$ \\
\hline $\begin{array}{l}\text { Length of } \\
\text { the trunk }\end{array}$ & & $\mathrm{x}$ \\
\hline $\begin{array}{l}\text { Thickness } \\
\text { of the trunk }\end{array}$ & & $\mathrm{x}$ & \\
\hline $\begin{array}{l}\text { Velocity of } \\
\text { the } \\
\text { assembly } \\
\text { line of the } \\
\text { unit }\end{array}$ & & $\mathrm{x}$ & \\
\hline $\begin{array}{l}\text { Feeding } \\
\text { rate of the } \\
\text { assembly } \\
\text { line of the } \\
\text { unit }\end{array}$ & & $\mathrm{x}$ & $\mathrm{x}$ \\
\hline $\begin{array}{l}\text { Type of } \\
\text { actuators } \\
\text { (cylinder) }\end{array}$ & $\mathrm{x}$ & $\mathrm{x}$ & \\
\hline $\begin{array}{l}\text { Number of } \\
\text { working } \\
\text { shifts } \\
\text { (1/2/3) }\end{array}$ & $\mathrm{x}$ & & \\
\hline
\end{tabular}

In an energy efficiency analysis of a woodworking plant, regular seasonal properties of the system must also be taken into account. These seasonal properties can be classified into short time and long-time nature. Typically they are periods when the assembly line is stopped.

Usually the working day consists of two 8-hour shifts. Depending on economic situation, the number of shifts may also be one or three. In case of two shifts, the pneumatic line is typically switched off in the night time (12p.m- 
8.a.m). Shorter breaks on regular basis typically occur on lunch time and during the change of shift.

In the long run, a longer standstill (of magnitude of 1 month) at the plant typically takes place on summer time. Also during public holidays, a break of a few days may take place.

Temporarily, the functioning of the assembly-line may stop due to blockages on the line. However, these breaks occur seldom and are short-time. Usually, on a woodworking plant, there are also reserve parts for defective actuators (cylinders).

\section{Measurements in compressed air systems}

During the last few years, measurements in several woodworking industrial plants in Finland have been made. The overall strategy of the measurements was to compare the energy consumption of different consumption network configurations by collecting data from one relatively long period - e.g. working with one configuration, repeat the same measurements with another configuration and compare results.

\subsection{Pressure measurements}

A time series of pressure values were measured. The measurements typically include pressure (bar) with sampling frequency of $1 \mathrm{~Hz}$.. The pressure measurement equipment consists of radio controlled data loggers. With SMS-commands, the measurement could be controlled remotely. These commands included start/stop/pause/continue qualities.

The most essential figures of the system are the maximum, minimum and average values of pressure. These values depend mostly on the pressure difference of the compressor. Since the measurement point is usually located behind the after-treatment system of the compressor, the losses cause that the measured values are typically $0.5-1$ bar lower than in the compressors outlet.

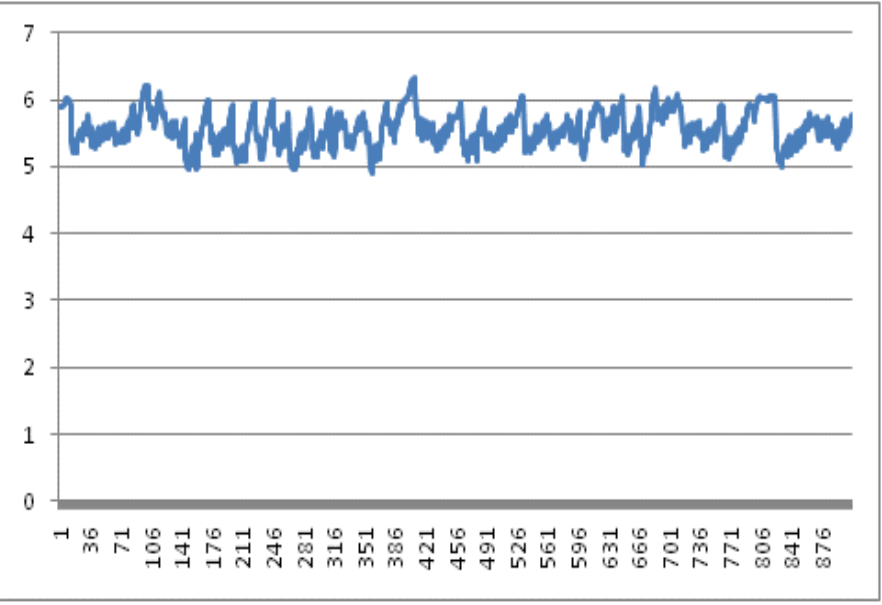

Figure 4: An example of pressure measurements(bars)
The measurements are usually performed at the consumption point. When the actual adjustable pressuredifference of the compressor is known, it is simple to get the pressure losses between production and consumption. However, in order to get the idea about the consumption type (pressure-dependent/independent consumption) the raw data requires some preparation.

Since compressors in many industrial applications usually work with on/off-basis, from the viewpoint of energy efficiency, the most essential factor is the proportion of the rise time of the total cycle time.

In some industrial applications, during the unloading period the compressor is idle running.

In a case, when a compressor runs with idle-power, the total energy can be obtained from (6) The idle running power of the motor is constant $P_{0}$ during the unloading period.

Total energy during the cycle is

$W_{t o t}=P^{\prime} t^{\prime}+P_{0}\left(t^{\prime \prime}-t^{\prime}\right)$

The average power during the period is respectively

$P_{\text {ave }}=\frac{t^{\prime}}{t^{\prime \prime}} P^{\prime}+\left(1-\frac{t^{\prime}}{t^{\prime \prime}}\right) P_{0}$

Typically $P_{0}$ is of magnitude $20-30 \%$ of the average power.

\subsection{Energy and power measurements}

Simultaneously with the pressure measurements, energy and power measurements were carried out. The frequency of the measurements was $1 / 30$ s. Since the sampling frequency is much slower than in pressure measurements, the results are not compatible. From the measurements, certain equivalencies between the potential variable (pressure), flow variable (mass flow) and energy/power consumption can be notified. For example, energy consumption is proportional to the production of compressed air (normal cubic meters). 


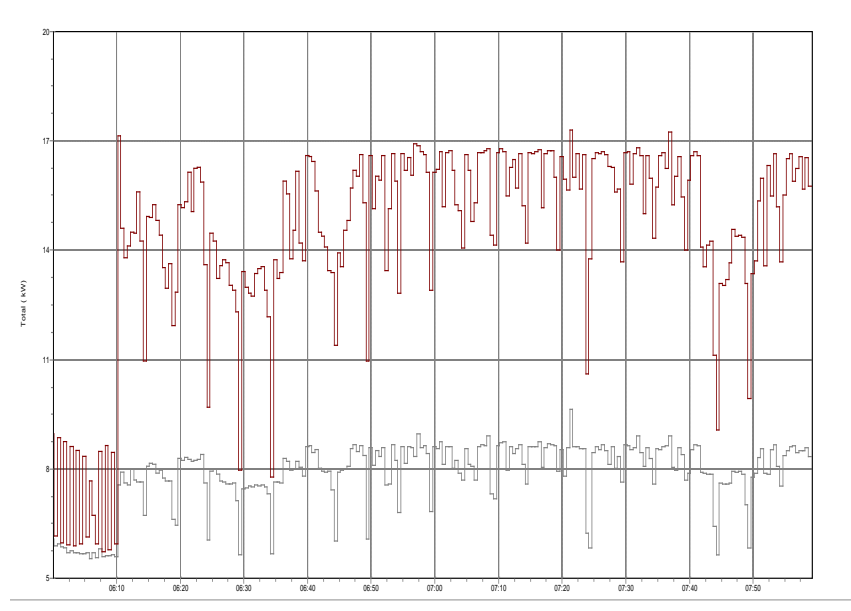

Figure 5: An example of power measurement.(maximum and average power consumption)

The quantities to be measured included minimum, maximum and average values of three-phase current, voltage, apparent and active power/energy in three phases and total. However, only minimum, maximum and average power are relevant from viewpoint of energy efficiency. In Fig.4 time series of minimum and average power consumption are presented. Since every measurement is discrete and the sampling frequency is high, the time series is not very smooth, but certain homogeneous behavior can be identified (maximum active power correspondence the nominal power of the compressor). In practice, it is more reasonable to examine the cumulative energy consumption (Fig.6,7), which can be directly integrated from the power.

\subsection{Interpretation of the measurements}

\subsubsection{Pressure measurements}

The measurement data of a two-point controlled compressor is in many applications typically noisy (Fig.4) A naturally arising question is whether periodicity in the pressure changes can be identified.

A standard widely-used filtering method for continuous time series is sliding average method. If the filtering method is successful, from the pressure time series the length of the total time cycle and the proportion of rise/fall time can be identified..

An autocorrelation plot of the pressure measurements during a reasonable long period e.g. one hour may be very informative (Fig.6).

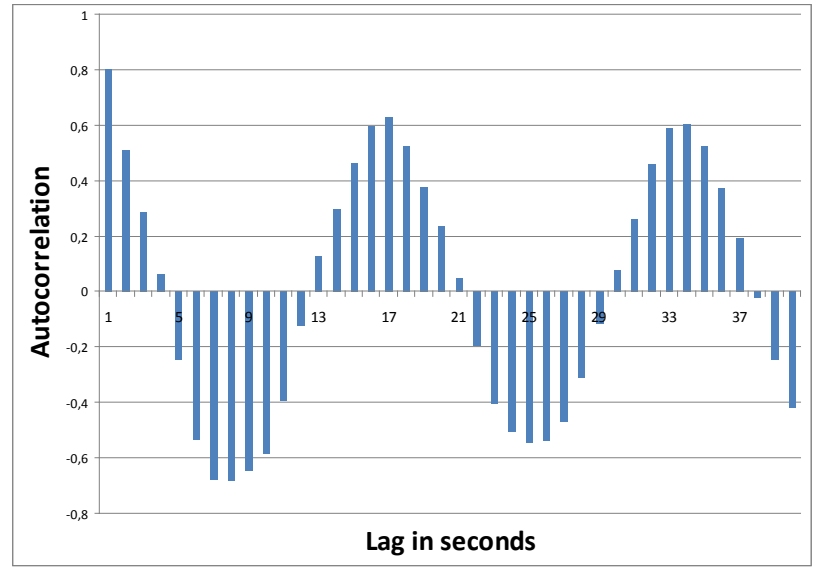

Figure 6: An example of an autocorrelation plot

For example, from figure 6, it can be inferred, that the pressure values between 15-20 seconds are quite strongly dependent. Combining the autocorrelation method with the approaching described in Ch. 3.2, the shape of the actual pressure curve can be estimated and proportion of the rise time (compressor on) of the total time determined.

\subsubsection{Energy and power measurements}

The trend of the cumulative energy consumption can be revealed by various regression methods [9].

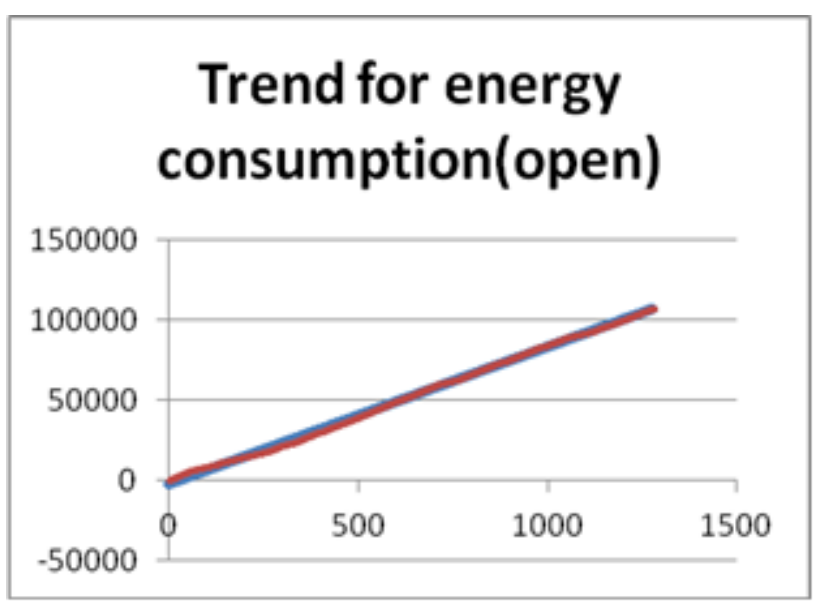

Figure 7: An example of an actual cumulative energy consumption (in $\mathrm{kWs}$ ) trend(red) and regression estimate for prediction (blue)

Figure 7 presents a measurement data of cumulative energy consumption from the period of one working day while a regression estimate based on a short time (1-2 hours) From the figure, one can make a conclusion, that energy consumption trend can be modeled with simple linear regression. 


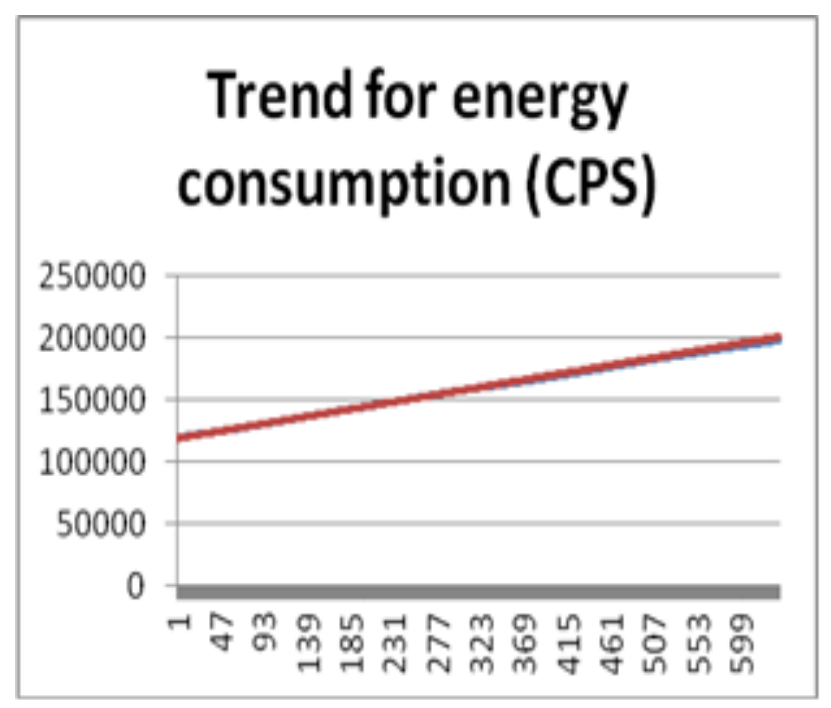

Figure 8: An example of an actual cumulative energy consumption trend(red) and regression estimate for prediction (blue)

The same energy/power measurements were repeated with different configuration on next day (Fig.7). The system in this measurement was CPS (Closed Pneumatic System), based on the recycling of CA [10]. Since the slope of the closed system is much lower than that of open system, the closed system evidently consumes less energy per time unit. From viewpoint of statistics, it is noteworthy that the trend remains linear even when the configuration is changed.

With certain reservations, one can generalize the results and make the normality assumption. In practice, the statistical properties affecting on the consumption of compressed air are often of mechanical reason and follow normal distribution. In that case, a confidence interval (95\%) for the energy consumption can be determined.

\section{Conclusions}

Since energy consumption of a compressor is strongly, almost linearly dependent on the consumption of CA, it is reasonable to analyze the energy efficiency issue by analyzing consumption points by their nature. In a typical manufacturing/assembly line process the most of the CA is used by actuators, the main actuator type being a doubleacting cylinder. From the physical principles and dimensions of the cylinder, the consumption of CA in a single consumption point can be determined analytically. The behavior of these consumptions is quite similar even for cylinders with different dimensions. According to the Central Limit Theorem, the sum of consumptions can be assumed to approach a normal distribution. Measurements of a relatively short time (one working day) can be generalized and a forecast of energy consumption with confidence interval be obtained.
In addition to regular consumptions, some consumptions on the manufacturing line, like blowing purposes are of stochastic nature. However their role is usually insignificant and has minor or no effect on the overall trend.

Furthermore, leaks have a permanent deteriorating effect on energy efficiency of industrial systems. Since the consumption of CA usually strongly depends on the pressure [7], the effect of the leaks on the overall energy efficiency becomes more evident when the pressure is high. In such CA systems, it is important to detect leaks in time. In practice, when the pressure measurements are performed during a stoppage when there is no natural CA consumption by keeping compressor on, even the proportion of leakage can be easily approximated from the pressure curve.

In practice, the energy consumption in an industrial pneumatic system is often a monotonic function, which can be approximated as a sum of a regression line and a normally distributed deviation component. In order to predict the energy consumption in the future an estimate in a certain confidence level can be calculated from the regression line combined with the stochastic component. The result is then ready to be used in energy saving calculations regarding the design of new pneumatic systems or the improvement of existing designs.

On workdays, from Monday to Friday, the consumption of CA typically follows some similar trend. However, on weekends, the trend may be quite different. Also, there may be certain seasonal components in the consumption of CA (e.g. in the summer the production in some branches of industry is lower than in the winter). Fitting seasonal components [9] to the overall consumption trend is a subject of further investigation.

Also, it is possible to model the CA system starting from first principle physical modeling, and then combining it with measurements added with stochastic component. The estimation of model parameters can be computed on-line by using the numeric model. The described approach opens new methods in energy saving techniques in the future. 


\section{Nomenclature}

\begin{tabular}{|c|c|c|}
\hline Designation & Denotation & Unit \\
\hline$\Phi(x)$ & Probability function & \\
\hline$\mu$ & Mean & \\
\hline$N$ & Number of cylinder movements & \\
\hline$p$ & Pressure & [bar] \\
\hline$p_{0}$ & Atmospheric pressure & [bar] \\
\hline$P$ & Nominal power & {$[\mathrm{kW}]$} \\
\hline$P_{\text {ave }}$ & Average power & {$[\mathrm{kW}]$} \\
\hline$P_{0}$ & Idle running power & {$[\mathrm{kW}]$} \\
\hline$\Pi$ & Relative pressure & \\
\hline$q_{i}$ & Consumption & {$\left[\mathrm{m}^{3} / \mathrm{min}\right]$} \\
\hline Q & Total consumption & {$\left[\mathrm{m}^{3} / \mathrm{min}\right]$} \\
\hline$r$ & Radius of the piston & {$[\mathrm{mm}]$} \\
\hline$R$ & Radius of the cylinder & {$[\mathrm{mm}]$} \\
\hline$S$ & Length of the impact & {$[\mathrm{mm}]$} \\
\hline$\sigma^{2}$ & Variance & \\
\hline$t_{c}$ & Cycle time & {$[\mathrm{s}]$} \\
\hline$t^{\prime}$ & Rise time (loading) & \\
\hline$t "$ & Fall time (unloading) & \\
\hline$W_{\text {tot }}$ & Total Energy & {$[\mathrm{kJ}]$} \\
\hline
\end{tabular}

\section{References}

[1] P Radgen, E Blaustein. (Eds) Compressed Air Systems in the European Union: Energy, Emission, Savings Potential and Policy Actions 2001

[2] The Energy Research Institute, Department of Mechanical Engineering, University of Cape Town How to save energy and money: Guide Book 3: Compressed Air Systems 2007

[3] Paul H Harris, Garret O’Donnell, Tim Whelan Energy Efficiency in Pneumatic Production Systems: State of the Art and Future Directions Leveraging Technology in a Sustainable World Proceedings of the 19th CIRP Conference on Life Cycle Engineering, University of California at Berkeley, Berkeley, USA 2012

[4] Paul H Harris, Garret O’Donnell, Tim Whelan. Predictive Consumption Models for Electro-Pneumatic Production Systems. IEEE Transactions on Mechatronics 2011

[5] Susanne V Krichel, O Savodny. Analysis and optimization of compressed air networks with model-based approaches, Ventil 17/2011

[6] Susanne V Krichel, O Savodny. Dynamic modeling of pneumatic transmission lines in MATLAB/SIMULINK Proceedings of the International Fluid Power and Mechatronics Conference, Beijing/China 2011

[7] J Parkkinen. Energy Efficiency in Industrial Pneumatic Systems Helsinki University of Technology Control Engineering Laboratory Espoo 2007 (lic.thesis)

[8] J Parkkinen, K Zenger. A new Efficiency Index for Analysing and Minimising Energy Consumption in Pneumatic Systems Int. Journal of Fluid Power2008

[9] G.E.P Box, G.M. Jenkins, G.C. Reinsel : Time Series Analysis: Forecasting and Control John Wiley \& Sons,Inc 2008

[10] J Parkkinen. Improving Energy Efficiency in Industrial Pneumatic Systems by Structural Means The Twelfth Scandinavian International Conference on Fluid Power 2011, Tampere, Finland

[11] V.Hölttä: Analysis of stem diameter measurement in forest harvester (diploma thesis)2004 